\title{
Protocolo de evaluación de la prosodia emocional y la pragmática en personas con esquizofrenia
}

\author{
Alfonso Martínez ${ }^{1}$, Manuela Martínez-Lorca², Jose Luis Santos ${ }^{3}$, y Alberto Martínez-Lorca ${ }^{4}$
}

Recibido: 13 de abril de 2018 / Revisado: 17 de mayo de 2018 / Aceptado: 31 de agosto de 2018

Resumen. La esquizofrenia se caracteriza por una distorsión del pensamiento, las percepciones, las emociones, el lenguaje, la conciencia de sí mismo y la conducta. Afecta a más de 21 millones de personas en todo el mundo y es una de las 10 enfermedades más incapacitantes según la Organización Mundial de la Salud, pero existe escasa evidencia sobre el déficit lingüístico con el que cursa la enfermedad. Estudio transversal y cuasi-experimental donde se analizan los datos resultantes de la evaluación por medio de diferentes pruebas de las áreas de pragmática y prosodia en una muestra compuesta por 96 sujetos, de los cuales 48 (50\%) no tienen enfermedad mental y componen el grupo control y 48 (50\%) tienen enfermedad mental y componen el grupo experimental.

De acuerdo con la clasificación de Crow (1978), el 56,7\% de los sujetos del grupo experimental (27 sujetos) tiene sintomatología positiva y el $43,8 \%$ (21 sujetos) tiene sintomatología negativa. Se observan diferentes patrones de déficit según la sintomatología, así como una afectación de las áreas evaluadas en el estudio (pragmática y prosodia afectiva).

La esquizofrenia cursa con déficit en las áreas analizadas, si bien existe una controversia acerca de su origen. En relación con la pragmática, encontramos interpretaciones literales en ambos tipos de pacientes, aunque son más frecuentes en los sujetos con sintomatología positiva. En cuanto a la prosodia, encontramos frecuencias fundamentales excesivas, monotonía en la expresión de las emociones y dificultades para comprender emociones a través de los aspectos prosódicos.

Palabras clave: esquizofrenia; lenguaje; evaluación; pragmática; prosodia emocional

\section{[en] Protocol for the evaluation of prosody and pragmatics in people with schizophrenia}

\footnotetext{
Abstract. Schizophrenia is characterized by a distortion of thinking, perceptions, emotions, language, self-awareness and behavior. It affects more than 21 million people worldwide and is one of the 10 most disabling diseases according to the World Health Organization, but there is little evidence of the language deficit entailed by the disease.

This is a cross-sectional and quasi-experimental study. A battery of different assessment instruments was used to evaluate pragmatics and prosody in a sample of 96 subjects, of which $48(50 \%)$ had no mental illness (control group) and 48 (50\%) had mental illness (experimental group).

1 Centro Crecer. Servicio de Atención Temprana (Toledo). España. manuela.martinez@uclm.es

2 Departamento de Psicología. Facultad de Terapia Ocupacional, Logopedia y Enfermería. Universidad de Castilla-La Mancha. España.

3 Psiquiatra Jefe del Área de Salud Mental. Hospital Virgen de la Luz (Cuenca). España.

4 Departamento de Anatomía y Embriología Humanas. Facultad de Medicina. Universidad Complutense de Madrid. España.
} 
According to the classification of Crow (1978), 56.7\% of the subjects of the experimental group (27 subjects) showed positive symptomatology and $43.8 \%$ (21 subjects) showed negative symptoms. Different deficit patterns were observed according to the symptomatology, as well as impairment of the areas assessed in the present study (pragmatics and affective prosody).

Schizophrenia entails a deficit in the analyzed areas, although there is a controversy about its origin. Concerning pragmatics, literal interpretations are to be found in both types of patients, but these are more frequent in subjects with positive symptomatology. As for prosody, the appearance of excessively high fundamental frequencies, monotony in the expression of emotions, and difficulties for the understanding of affective prosody are the most salient characteristics of all the patients in the experimental group.

Key words: schizophrenia; language; assessment; pragmatics; affective prosody

Cómo citar: Martínez, A., Martínez-Lorca, M., Santos, J.L., y Martínez-Lorca, A. (2018). Protocolo de evaluación de la prosodia emocional y la pragmática en personas con esquizofrenia. Revista de Investigación en Logopedia, 8(2), 129-146.

\section{Introducción}

La esquizofrenia se define como un trastorno psicótico crónico que incluye alteraciones conductuales, afectivas y cognitivas (American Psychiatric Association, 2013). Afecta aproximadamente al 1\% de la población mundial (OMS, 2016; Ritsner y Gottesman, 2011). La sintomatología de la esquizofrenia se divide en síntomas positivos y negativos. Los síntomas positivos son definidos como aquellas conductas y cogniciones caracterizadas por alucinaciones, delirios, conductas extravagantes, etc. Los síntomas negativos consisten en conductas y cogniciones que están presentes en la población general, pero disminuidas o ausentes en pacientes con esquizofrenia como, por ejemplo, el aplanamiento afectivo, anhedonia, apatía/indiferencia, etc. (Crow 1997; National Institute of Health, 1992).

Frecuentemente se relaciona la esquizofrenia con disfunción social y pobre adaptación psicosocial, lo que afecta a diferentes aspectos de la vida del paciente (Knapp, Mangalore y Simon, 2004; Mondragón-Maya, Ramos-Mastache, Román y Yáñez-Téllez, 2017). Además, se ha reportado consistentemente que los pacientes con esquizofrenia muestran deterioro cognitivo, particularmente en atención, memoria, velocidad de procesamiento, funcionamiento ejecutivo, destreza motora, atención sostenida, lenguaje y funciones ejecutivas (solución de problemas, flexibilidad mental y planeación) (Mesholam-Gately, Giuliano, Goff, Faraone y Seidman, 2009; Napal et al., 2012). Este deterioro cognitivo progresa a medida que lo hace la enfermedad.

El déficit lingüístico asociado a la esquizofrenia se manifiesta en un discurso desorganizado e incoherente, unido a dificultades anómicas, de comprensión, así como para la expresión y comprensión de emociones expresadas a través del lenguaje. No hay acuerdo en la etiología de estos problemas, que han sido atribuidos bien a un fallo en las funciones ejecutivas (Baddeley, 1993), en especial en la memoria operativa y la atención (Cavieres y Valdebenito, 2005; Pardo, 2005), bien a un déficit primordialmente lingüístico (Chapman, Chapman y Miller, 1964; Crow, 1997; Faber, Abrams, Taylor, Kasprison, Morris y Weisz, 1983; Kleist, 1960; Rochester, 1978; Wróbel, 1990). 
En concreto, las áreas lingüísticas más afectadas en la esquizofrenia son la semántica (Berberian et al., 2016; Kubicki, 2003) y, especialmente, la pragmática (Martínez, Felizzola y Matallana, 2015; Salavera y Puyuelo, 2010), insistiendo en la conservación parcial de la sintaxis y la morfología (Covington et al., 2005; DeLisi, 2001), ya que, aunque esta pueda aparecer desestructurada en los discursos, la morfosintaxis se mantiene correcta.

Concretamente, en el área de pragmática se ha puesto de manifiesto que las personas con esquizofrenia presentan dificultades con los pares adyacentes (Durán y Figueroa, 2009), la coherencia, la cohesión y el mantenimiento del tema (Faber y Reichstein, 1981). Presentan también menor diversidad léxica y menor claridad, así como conexiones débiles en el discurso (Belinchón, 1988a; Insúa, Grijalvo y Huici, 2001).

Además, numerosas investigaciones han señalado que el déficit en la prosodia emocional es patognomónico de la esquizofrenia (Beaucousin et al., 2006; Hoekert, Kahn, Pijnenborg y Aleman, 2007; Leentjens, Wielaert, van Harskamp y Wilmink, 1998; Martínez-Sánchez et al., 2015; Murphy y Cutting, 1990). Las cualidades prosódicas del habla son determinadas por tres parámetros (duración, intensidad y tono de los sonidos del habla) que afectan tanto al significado de lo que se dice, como a las emociones expresadas.

Así, pueden distinguirse una prosodia lingüística, que cumple un papel similar al de la puntuación en los textos escritos y que determina la modalidad oracional, y una prosodia emocional o afectiva, que se genera cuando el hablante expresa contenidos afectivos en el mensaje, y que es la que se encuentra afectada en los pacientes con esquizofrenia (Martínez-Sánchez et al., 2015). La mayoría de investigaciones solo se ocupan de la compresión de la prosodia lingüística (Hoekert et al., 2007), dejando de lado la capacidad de expresión de emociones a través de la prosodia. Es importante señalar que el procesamiento de la prosodia emocional se localiza en el lóbulo temporal derecho (Beaucousin et al., 2006), área cerebral afectada en la esquizofrenia. En efecto, el embotamiento afectivo es el síntoma que goza de mayor aceptación como característica clínica del trastorno, y parece ser mayor en pacientes con sintomatología negativa, aunque existe controversia al respecto (Pascual, Solé, Castillón, Abadía y Tejedor, 2005).

En cualquier caso, las personas afectadas de esquizofrenia presentan alteraciones en diversas áreas lingüísticas, lo que hace necesaria su evaluación (Durán y Figueroa, 2009; Insúa et al., 2001). Para la evaluación lingüística de estos pacientes resulta útil usar el enfoque de la Lingüística Clínica Funcional (LCF), que focaliza su interés en la descripción detallada de la conducta verbal y comunicativa de los hablantes con déficit, y no en la determinación de su etiología (Perkins y Howard, 2011). El paradigma de la LCF señala también la importancia de reunir evidencia del perfil comunicativo de patologías mentales o neurológicas graves dentro de los parámetros del trabajo clínico con pacientes psiquiátricos. Por último, la LCF asume tres presupuestos teóricos (Gallardo-Paúls y Hernández-Sacristán, 2013): 1) que no se puede separar el lenguaje de las dimensiones sociales y cognitivas del individuo que lo produce; 2) que el sistema lingüístico no se puede separar de los usos lingüísticos a los que sirve, y 3) que el lenguaje rehabilita el lenguaje (Estabrooks y Albert, 2004). Por ello se tendrán en cuenta tanto los aspectos preservados como los deficitarios del discurso de los pacientes. 
De este modo, la evaluación del déficit lingüístico en la esquizofrenia es un paso previo fundamental para tomar las medidas más oportunas y adecuadas de cara a la rehabilitación lingüística. Por todo ello, el objetivo de la presente investigación será la evaluación de las dimensiones pragmática y prosódica en los pacientes con esquizofrenia. En el nivel pragmático se evaluarán las siguientes categorías: pares adyacentes, interpretaciones literales, coherencia, cohesión y mantenimiento del tema, mientras que las categorías evaluadas en el nivel prosódico serán la capacidad de comprensión y expresión de emociones a través de la voz . La hipótesis de partida es que las personas con esquizofrenia obtendrán peores resultados en ambos niveles de evaluación que las personas sin este trastorno mental.

\section{Metodología}

\section{Muestra}

Se trata de un estudio transversal y cuasi-experimental con una muestra de 96 sujetos de los cuales 48 (50\%) tienen esquizofrenia y componen el grupo experimental, y 48 $(50 \%)$ no tienen enfermedad mental, constituyendo el grupo control. Se decidió dividir el grupo experimental según la sintomatología, ya que la afectación lingüística es diferente según los síntomas predominantes de la enfermedad (APA, 2013): los pacientes con sintomatología negativa se caracterizan por un embotamiento afectivo que afecta a la diversidad léxica, y los pacientes con sintomatología positiva se caracterizan por las alucinaciones que afectan a la coherencia del discurso. El grupo experimental está compuesto por 39 sujetos crónicos y 9 en los primeros estadios de la enfermedad.

Se tuvieron en cuenta los siguientes criterios diagnósticos para el grupo de personas con esquizofrenia: a) diagnóstico de esquizofrenia según los criterios del DSM 5 (APA, 2013); b) no tener diagnósticos asociados o subtipos de la esquizofrenia como trastorno esquizoafectivo; c) que pasara más de un año y medio desde que se produjo el diagnóstico para evitar falsos positivos y d) presentar sintomatología positiva/ negativa en el momento del reclutamiento.

El reclutamiento se llevó acabo en diferentes asociaciones de salud mental, como por ejemplo, AMAFE (Asociación Madrileña de Familiares y Amigos de Personas con Esquizofrenia), la Asociación VIVIR (Cuenca), el centro de Rehabilitación Psicosocial de Tomelloso (Ciudad Real) y ATAFES de Talavera de la Reina (Toledo). También colaboró la Unidad de Salud Mental del Hospital Virgen de la Luz (Cuenca). Los participantes del grupo control fueron personas de diferentes poblaciones de las Comunidades Autónomas de Madrid, Ciudad Real, Cuenca y Toledo, sin estar emparejados con respecto a los sujetos del grupo experimental en ninguna de sus características.

Tras ser informados detalladamente del objetivo y procedimiento del estudio, se solicitó a los participantes la firma de un consentimiento informado en el que se garantiza su anonimato (según se recoge en la Ley Orgánica 15/1999, de 13 de diciembre, de Protección de Datos de Carácter Personal) y se recoge la posibilidad de revocar dicho consentimiento en cualquier momento. 


\section{Instrumentos}

Para la evaluación lingüística de los pacientes con esquizofrenia encontramos varias dificultades. Existen escasos instrumentos baremados y pruebas estandarizadas que evalúen las capacidades lingüísticas de estos pacientes. Algunos test como SAPS/ SANS (Andreasen, 1984) las evalúan, pero de manera subjetiva. En otros casos, como el test de COTWAN (Controlled Oral Word Association Test, de Benton y Hamsher, 1989) se usan fonemas para acceder al léxico del inglés, lo cual no es funcional en castellano y no permite generalización de los resultados. En definitiva, existen pocas opciones de evaluación del lenguaje de la población con psicosis en español, por lo que se optó por la construcción de una batería de evaluación de los aspectos pragmáticos y prosódicos.

Los instrumentos de medida consistieron, por un lado, en un cuestionario elaborado ad hoc para conocer datos sociodemográficos, antecedentes familiares, historia de la enfermedad, tipo de medicación, edad del primer brote psicótico, cuestionas laborales, etc. Por otro lado, se utilizaron escalas que evalúan la dimensión pragmática y prosódica del lenguaje, adaptándolas a la población objeto de estudio. Para la evaluación de la dimensión pragmática se utilizaron los siguientes instrumentos:

- El apartado de interpretación de metáforas de la versión en español del Protocolo MEC (Batería de Montreal de Evaluación de la Comunicación) (Abusamra, Martínez Cuitiño, Molina y Ferreres, 2006). Este apartado consiste en leer un total de 20 enunciados donde el sujeto tiene que elegir, entre tres opciones posibles, la interpretación más adecuada para cada enunciado leído.

- El PREP-R (Protocolo Rápido de Evaluación Pragmática-Revisado) (Fernández-Urquiza, Díaz, Moreno, Lázaro y Simón, 2015) ya que nos permite tener un valor cuantificable para la pragmática general, la habilidad pragmática específica (habilidades inferenciales, capacidad metarrepresentacional y conciencia metapragmática) y la habilidad pragmática de base gramatical (el correcto funcionamiento de los componentes fonológico, morfológico, sintáctico y semántico del lenguaje). La evaluación de estos aspectos se realizó por medio del análisis de la conversación libre que se tuvo al principio con todos los pacientes, la entrevista semiestructurada y la conversación dirigida a través de la lámina del test de Boston (Goodglass y Kaplan, 1992).

Dentro de la pragmática un elemento lingüístico importante es la evaluación de otros tipos de discurso además del conversacional, para lo que se utilizaron las siguientes pruebas:

La conocida lámina del Test de Boston "el robo de las galletas" (Goodglass y Kaplan, 1992). Se le pedía a los sujetos que, tras mirar detenidamente la lámina, describieran lo que ven con el objetivo de evaluar sus habilidades descriptivas.

La "Historia del Corsario Landolfo Rufolo" (Belinchón, 1986; Insúa et al., 2001; Van Dijk, 1980). En este caso, los pacientes tenían que intentar resumir la historia que previamente se les había leído. Los criterios para la evaluación de la historia escrita son los propuestos por Gómez (1976), que evalúan indirectamente las capacidades pragmáticas de base gramatical, puesto que se trata de categorías que influyen notablemente en la coherencia y cohesión del texto. Estos criterios son:

- El CAV o cociente entre adjetivos y verbos (Frostig, 1940). Si este índice es bajo indica una carencia de calificativos de descripciones en el discurso, acompañado de mucha acción; si el índice es alto, se interpretará en sentido opuesto. 
- TTR (Type Token Ratio) es una medida léxico métrica que evalúa el grado de heterogeneidad, diversidad y flexibilidad léxica del habla. Se calcula, simplemente, dividiendo el número total de palabras distintas que componen un texto, entre el número total de palabras que componen el mismo. Sus valores oscilan por tanto entre el 0 y el 1 siendo los valores más bajos los que reflejan mayor grado de perseverancia. Hay que tener en cuenta que, a medida que aumenta la longitud del discurso, la TTR tiende a bajar, porque necesariamente hay muchas palabras funcionales que se repiten. Si esto es así sucedería indistintamente tanto en el grupo control como en el grupo experimental, mientras que si las diferencias son estadísticamente significativas, ello indicaría una particular dificultad en los sujetos con esquizofrenia.

Para la evaluación de la dimensión prosódica, los pacientes escucharon una grabación en la que una actriz de teatro reproduce la frase de contenido lingüístico emocionalmente neutro "en invierno las noches son largas y los días cortos" (Pascual et al., 2005), en los cinco estados emocionales (LeDoux y Bernal, 1999) que son: alegría, enfado, tristeza, miedo y sorpresa, así como en las emociones vergüenza, culpa, admiración, asco (Scherer, 1979). La consigna proporcionada fue "Ahora va a escuchar a una actriz diciendo la frase, en invierno las noches son largas y los días cortos, la va a decir como si estuviera enfadada, alegre, triste, con culpa, con admiración, con asco, con miedo, con vergüenza y sorprendida. Cuando escuche la frase rodee en el siguiente papel cómo cree que se siente y ponga un uno para la primera grabación, un dos para la segunda y así con todas. Piense que no se repiten los estados de ánimo".

Por último, para la evaluación de la prosodia emocional pedimos a los pacientes que dijeran la misma frase "en invierno las noches son largas y los días cortos" en los diferentes estados de ánimo anteriores. Para la evaluación de los parámetros acústicos de la voz se empleó el programa PRAAT, cuantificándose los parámetros de frecuencia fundamental (expresada en hercios), velocidad (número de palabras por minuto) e intensidad (expresada en decibelios) que se asocian a cada emoción según distintos estudios (Francisco, Hervás y Gervás, 2005; Rodríguez et al., 1998). Para una cuantificación más detallada se emplearon los criterios del estudio de Scott (1983):

\begin{tabular}{|c|c|c|}
\hline \multicolumn{2}{|c|}{ Puntuaciones de la prosodia anormal } & Puntuaciones \\
\hline Volumen & Normal. Ruidoso, Bajo, flojo & \multirow{7}{*}{$\begin{array}{l}\text { 0 Normal } \\
\text { 1: } \text { Otro tipo de } \\
\text { medida }\end{array}$} \\
\hline Frecuencia & Normal, Alta, baja, monótona, rota & \\
\hline Tono & Normal, hipertono, hipotono, mixto & \\
\hline Entonación & $\begin{array}{l}\text { Normal, monótona, reducida, } \\
\text { inapropiada }\end{array}$ & \\
\hline Calidad Vocal & $\begin{array}{l}\text { Normal, estridente, ronco. } \\
\text { Temblorosa, afónico }\end{array}$ & \\
\hline Ritmo & $\begin{array}{l}\text { Normal. tartamudo, silencios } \\
\text { inapropiados, insuficiente velocidad. }\end{array}$ & \\
\hline Velocidad & $\begin{array}{l}\text { Normal. muy rapido, aumentado } \\
\text { seguin se va hablando, decreciendo } \\
\text { segun se va hablando| }\end{array}$ & \\
\hline
\end{tabular}




\section{Procedimiento}

El presente trabajo fue presentado al Comité Ético del Hospital Universitario Virgen del Prado (Talavera de la Reina, Toledo), del que recibió un dictamen favorable (código 11/2017). A partir de ese momento se inició el contacto con las asociaciones y la Unidad de Salud Mental del Hospital Virgen de la Luz (Cuenca) para proponer un cronograma de trabajo para la recogida de datos, que tuvo lugar en los meses de mayo y junio de 2017.

Con anterioridad a la recogida de datos, el responsable de la investigación realizó un pilotaje con cuatro pacientes de la asociación AMAFE para comprobar la carga cognitiva de la prueba, la sobrecarga atencional, el orden y la dificultad de los ejercicios. Una vez realizadas las modificaciones oportunas en función de los resultados obtenidos en el pilotaje, la prueba quedó compuesta por los instrumentos anteriormente descritos, y dio comienzo la recogida de datos.

El análisis estadístico de los datos se realizó con el programa informático IBM ${ }^{\circledR}$ SPSS ${ }^{\circledR}$ Statistics 22.0. En primer lugar, se comprobó si las variables seguían una distribución de normalidad mediante la prueba Kolmogorov-Smirnov (K-S) de normalidad.

La prueba K-S determinó que la muestra no sigue una distribución de datos normal, sino que todas las variables evaluadas siguen una probabilidad menor o igual 0,05 . Por lo tanto, para el análisis de los datos se realizó la prueba no paramétrica de Mann-Whitney que es la prueba no paramétrica paralela a la prueba $t$ para muestras independientes. Se ha tenido en cuenta para todos los análisis estadísticos un nivel de confianza del .05. Además, se utilizaron distribución de frecuencias y descriptivos (principalmente, medias y desviaciones típicas) y pruebas de independencia Chi-cuadrado.

\section{Resultados}

En la Tabla 1 pueden verse los datos más relevantes de las características sociodemográficas del presente estudio.

Tabla 1. Datos sociodemográficos

\begin{tabular}{|l|l|l|l|}
\hline Características & G.E. (\%) & G.C. (\%) & N. (\%) \\
\hline Género & & & \\
\hline Hombre & $32(66,7 \%)$ & $25(52,1 \%)$ & $57(59,4 \%)$ \\
\hline Mujer & $16(33,3 \%)$ & $23(47,9 \%)$ & $39(40,6 \%)$ \\
\hline Edad (M, SD) & $\begin{array}{l}45,56 \text { años con una } \\
\text { desviación están- } \\
\text { dar de } 16,61\end{array}$ & $\begin{array}{l}43,34 \text { años con } \\
\text { una desviación } \\
\text { estándar de } 14,02\end{array}$ & $41,86($ SD 15,03) \\
\hline Trabajan & $3(6,3 \%)$ & $23(47,9 \%)$ & $26(27,1 \%)$ \\
\hline Sí & $45(93,8 \%)$ & $25(52,1 \%)$ & $70(72,9 \%)$ \\
\hline No & & & \\
\hline Estudios & & & \\
\hline
\end{tabular}




\begin{tabular}{|c|c|c|c|}
\hline Primarios & $17(35,4 \%)$ & $21(43,8 \%)$ & $38(39,6 \%)$ \\
\hline Secundarios & $19(39,6 \%)$ & $5(10,4 \%)$ & $24(25 \%)$ \\
\hline Superiores & $12(25 \%)$ & $22(45,8 \%)$ & $34(35,4 \%)$ \\
\hline \multicolumn{4}{|l|}{ Estado civil } \\
\hline Soltero & $46(95,8 \%)$ & $20(41,7 \%)$ & $66(68,8 \%)$ \\
\hline Separado & $1(2,1 \%)$ & $13(27 \%)$ & $14(14,6 \%)$ \\
\hline Pareja de hecho & $1(2,1 \%)$ & $9(19 \%)$ & $10(10,4 \%)$ \\
\hline Otro & $0(0 \%)$ & $6(12,3 \%)$ & $6(6,2 \%)$ \\
\hline \multicolumn{4}{|l|}{ Actividades físicas } \\
\hline Sí & $33(68,6 \%)$ & $16(33,3 \%)$ & $49(51 \%)$ \\
\hline No & $15(31,4 \%)$ & $32(66,7 \%)$ & $29(30,2 \%)$ \\
\hline \multicolumn{4}{|l|}{ Consumo } \\
\hline Sí & $26(54,6 \%)$ & $27(56,2 \%)$ & $53(44,8 \%)$ \\
\hline No & $22(45,4 \%)$ & $21(43,8 \%)$ & $43(55,2 \%)$ \\
\hline \multicolumn{4}{|l|}{ Sintomatología } \\
\hline Positiva & $27(56,7 \%)$ & 0 & $54(56,7 \%)$ \\
\hline Negativa & $21(43,8 \%)$ & 0 & $42(43,3 \%)$ \\
\hline \multicolumn{4}{|c|}{$\begin{array}{l}\text { Desencadenante de los } \\
\text { brotes psicóticos: }\end{array}$} \\
\hline Divorcio & $1(2,1 \%)$ & 0 & \\
\hline Consumo & $3(8,4 \%)$ & 0 & \\
\hline Grandes problemas & $18(37,5 \%)$ & 0 & \\
\hline Estrés & $3(6,3 \%)$ & 0 & \\
\hline No lo sabe & $22(45,8 \%)$ & 0 & \\
\hline Enfermedad física & $1(2,1 \%)$ & 0 & \\
\hline \multicolumn{4}{|c|}{$\begin{array}{l}\text { Antecedentes familiares } \\
\text { de enfermedad mental: }\end{array}$} \\
\hline Sí & $28(58,3 \%)$ & $3(3,7 \%)$ & $31(31,2 \%)$ \\
\hline No & $19(39,6 \%)$ & $45(96,3 \%)$ & $64(66,7 \%)$ \\
\hline No sabe & $1(2,1 \%)$ & 0 & $1(2,1 \%)$ \\
\hline
\end{tabular}

Con respecto al consumo de sustancias, el 54,6\% del grupo experimental (26 sujetos) no ha consumido tóxicos en los meses previos al brote psicótico, provocando que no haya una relación significativa de consumo y enfermedad mental, de esta manera se descartaron patologías duales.

Por último, observamos que existe una gran variedad en la medicación de los sujetos, encontrando medicamentos con efectos sedativos, anticolinérgicos, de bloqueo $\infty$-adrenérgico y con efectos extrapiramidales. De nuestra muestra el medicamento más consumido ( 9 sujetos) es Leponex en distintas cantidades. El resto de los sujetos toman distintos medicamentos y en distinta cantidad, en función de la sintomatología de la enfermedad, así como de la cronicidad de esta. 
Los resultados del área lingüística de la pragmática (ver gráfica 1 y 2) reflejan relaciones significativas entre los grupos en los resultados totales de prueba de interpretación de metáforas del Protocolo $\operatorname{MEC~}(\mathrm{z}=-7,003 ; \mathrm{p} \leq 0,000)$, con un rango promedio para el grupo control de 65,50 y de 31,50 para el grupo experimental. Se encuentra también una correlación positiva entre las expresiones idiomáticas y las metáforas $\left(\chi^{2}(56)=103,865 ; \mathrm{p} \leq 0,000\right)$ con peores resultados para las idiomáticas. Las expresiones idiomáticas son expresiones lexicalizadas, es decir, secuencias de palabras con un significado unitario que no se deriva del de sus componentes, y que, por tanto, no responde al Principio de Composicionalidad. Sin embargo, las personas con esquizofrenia solo eran capaces de entender la interpretación literal que se derivaría de la aplicación de este principio, y no la figurada. Por lo que respecta a las metáforas, consisten en denominar, describir o calificar un término A través de su semejanza o analogía con un término $\mathrm{B}$, y por lo tanto para su interpretación se debe conocer la relación entre los conceptos que ambos términos designan. Las personas con esquizofrenia también mostraron dificultades en este sentido, aunque en menor medida que en la interpretación de expresiones idiomáticas. A modo de ejemplo, una de las respuestas propuesta por los sujetos experimentales cuando se les preguntaba el significado de la expresión metafórica la cocina está hecha una pocilga, era que en el final de la cocina había una pocilga.

También aparecen relaciones significativas en todos los parámetros evaluados por el Protocolo Rápido de Evaluación Pragmática Revisado: para la habilidad pragmática general $(\mathrm{z}=-8,911 ; \mathrm{p} \leq 0,000)$ con un rango promedio en el grupo experimental de 24,60 y de 72,40 para el grupo control; para la habilidad pragmática específica $(\mathrm{z}=-8,826 ; \mathrm{p} \leq 0,000)$ con un rango promedio de 27,30 en el grupo experimental y de 79,23 en el grupo control; y para la pragmática de base gramatical $(z=-8,830 ; p$ $\leq 0,000$ ) con un rango promedio en el grupo experimental de 24,89 y de 72,11 en el grupo control.

Los resultados de la lámina del Test de Boston, al ser variables categóricas, fueron analizados con la prueba chi-cuadrado de Pearson, apareciendo resultados significativos en todos los parámetros evaluados. Así, se encontraron parafasias fonéticas $\left(\chi^{2}(1)=12,080 ; p \leq 0,001\right)$ en el $88,2 \%$ de los sujetos del grupo experimental, frente al $11,8 \%$ de los sujetos del grupo control. En cuanto a las parafasias semánticas $\left(\chi^{2}(1)=28,611 ; \mathrm{p} \leq 0,000\right)$ también se dan en el $96 \%$ de los sujetos del grupo experimental y en el $4 \%$ de los sujetos control. La anomia $\left(\chi^{2}(1)=24,403 ; p \leq 0.000\right)$ se da en el $89,3 \%$ de los sujetos del grupo experimental y en un $10,7 \%$ de los sujetos control. Las repeticiones $\left(\chi^{2}(1)=15,590 ; \mathrm{p} \leq 0,000\right)$ se dieron en un $74,4 \%$ de los discursos de los sujetos experimentales y en un $25,6 \%$ del grupo control. Por último, los elementos que no aparecen en la lámina $\left(\chi^{2}(1)=41,552 ; \mathrm{p} \leq 0,000\right)$ se dieron en $100 \%$ de los discursos de los sujetos experimentales y en ninguno de los del grupo control. Así, encontramos que los pacientes con esquizofrenia, especialmente aquellos que cursan la enfermedad con sintomatología positiva, son los que producen un mayor número de elementos aberrantes en la Lámina de Boston (así, por ejemplo, uno de los sujetos indica que el agua se sale por un maleficio; otro dice que hay unos niños en el parque de fuera pasándoselo bien; otros señalan que los niños están cogiendo una tarta - lo que podría ser bien una parafasia semántica, o bien una invención de los propios sujetos). A la falta de conexidad e incoherencia del discurso contribuye también el uso de frases inconexas, la escasez de palabras funcionales, y el exceso de circunloquios. El habla de la mayoría de los pacientes era entrecortada 
y muchas veces ininteligible, dificultades articulatorias que podrían ser provocadas como efecto secundario de los antipsicóticos.

Por último, los resultados de la prueba escrita de la "Historia del Corsario Landolfo Rufolo" ponen de manifiesto relaciones significativas tanto en los parámetros del CAV como en los del TTR.

Así, analizados los resultados de la medida del CAV (cociente entre adjetivos y verbos), encontramos relaciones significativas entre los grupos evaluados a través de la prueba no paramétrica de Mann-Whitney $(\mathrm{z}=-6,745 ; \mathrm{p} \leq 0,000)$ con un promedio de 26,91 para el grupo experimental y un promedio de 64,46 para el grupo control. Igualmente, se obtuvieron relaciones significativas para la medida TTR (cociente entre palabras diferentes y palabras totales del texto) $(z=-2,472 ; p \leq 0,013)$ con un promedio de 39,32 en el grupo experimental y uno de 53,08 en el grupo control. Hay que indicar que cuatro sujetos del grupo experimental no fueron capaces de escribir nada ya que declaraban no recordar nada. Se incluye un fragmento de escritura de una paciente a modo de ilustración (Figura 1).

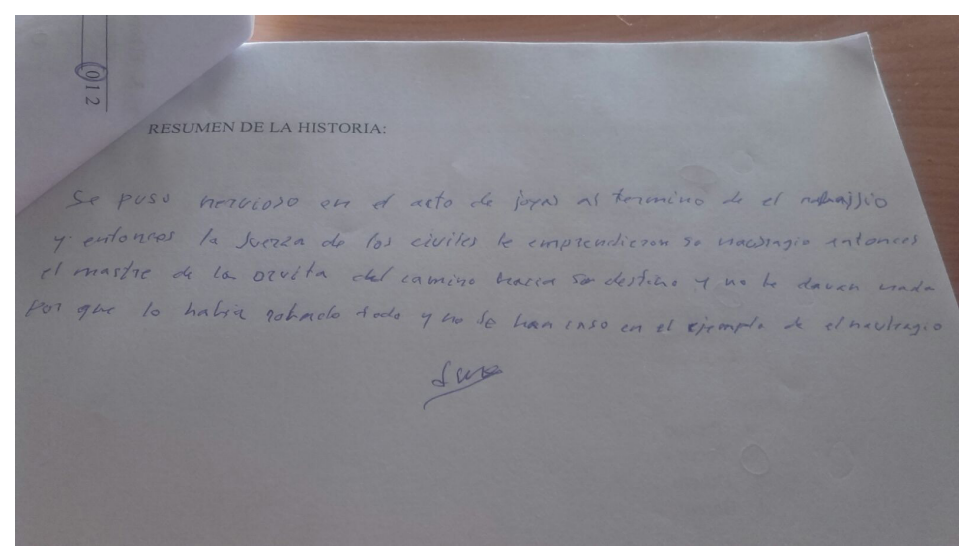

Figura 1. Ejemplo ilustrativo de escritura en la Historia del Corsario Landolfo Rufolo

En cuanto a las puntuaciones en la evaluación de los parámetros acústicos de la prosodia (ver gráfica 1 y 2) pone de manifiesto como la intensidad no tiene diferencias significativas entre los grupos, pero sí que los tienen la frecuencia fundamental y la velocidad de habla. La frecuencia fundamental tiene relación significativa entre los grupos $(z=-3,129 ; p \leq 0,002)$ con un rango promedio de 57,40 para el grupo experimental y un rango promedio de 39,60 para el grupo control.

También se encuentra una relación significativa en el parámetro acústico de la voz de velocidad por minuto $(z=-2,184 ; p \leq 0,029)$ con un rango medio para el grupo experimental de 42,29 y de 54,71 para el grupo control.

La evaluación de la prosodia comprensiva y expresiva es significativa entre los grupos siendo para la prosodia comprensiva $(\mathrm{z}=-7,517 ; \mathrm{p} \leq 0,000)$ con un promedio de 26,68 en el grupo experimental y de 68,88 en el grupo control. Y para la prosodia expresiva $(z=-7,697 ; p \leq 0,000)$ con promedios de 26,68 y 68,88 en el grupo experimental y en el grupo control, respectivamente. 


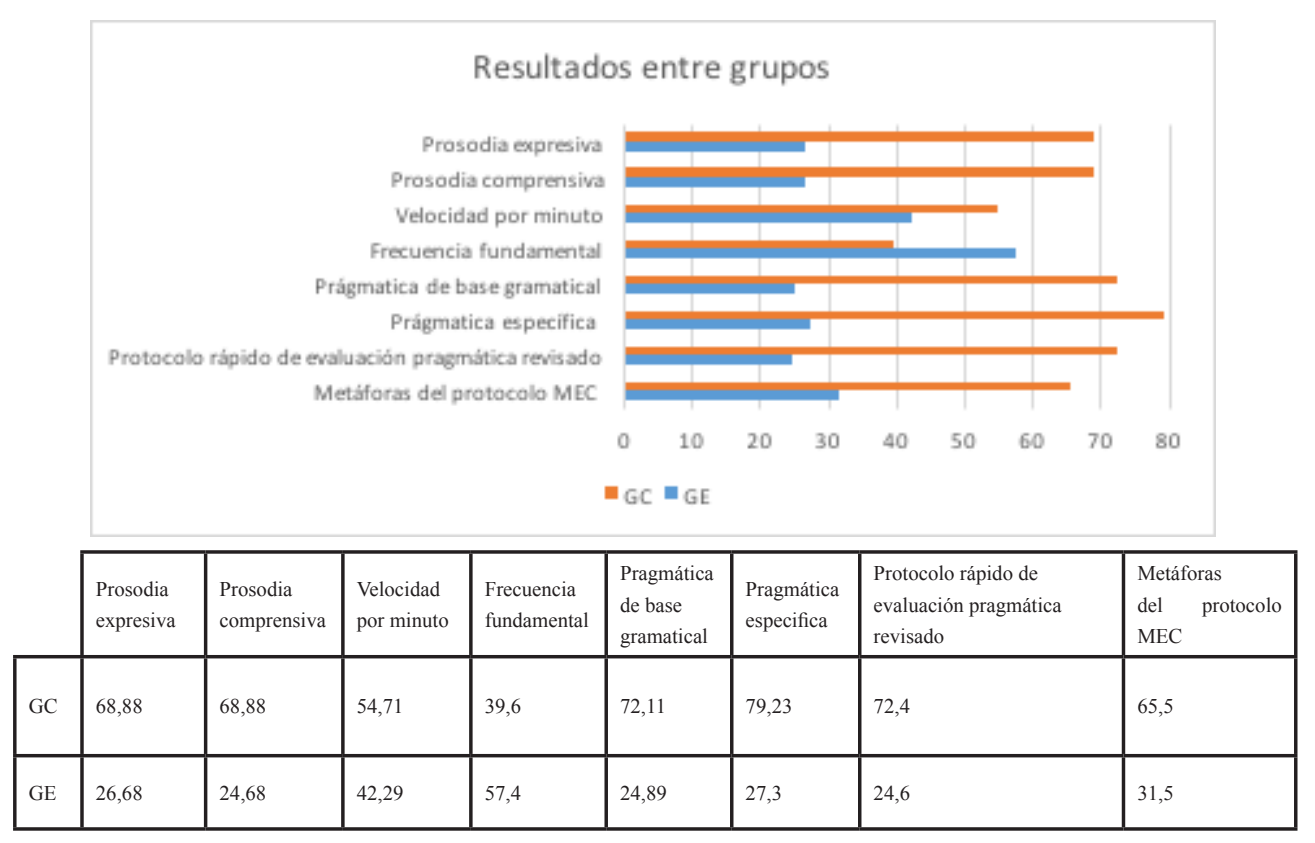

Gráfica 1: Resultados de las distintas pruebas de pragmática y prosodia

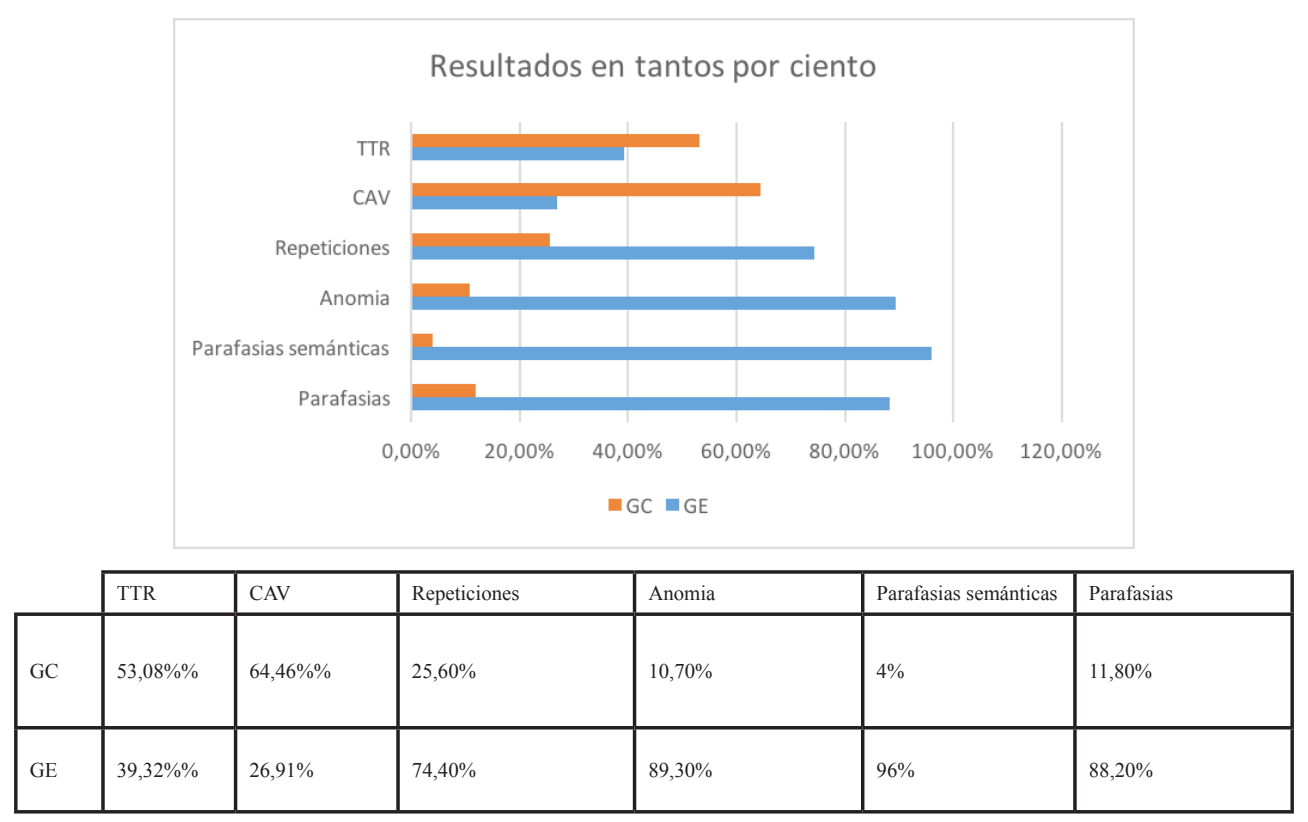

Gráfica 2: Resultados de las distintas pruebas de pragmática y prosodia 


\section{Discusión}

En primer lugar, los resultados de nuestras variables sociodemográficas ponen de manifiesto que casi tres tercios de la muestra no trabaja, mientras un tercio tiene estudios universitarios, aunque indicaron tardar más tiempo en obtenerlos, posiblemente por el déficit cognitivo con el que cursa la enfermedad (Cavieres y Valdebenito, 2005), o solo cursaron los primeros años hasta que se produjo el primer brote psicótico. Las personas con esquizofrenia se enfrentan al estigma social provocado por el desconocimiento de la enfermad, así como a los prejuicios y/o estereotipos sobre la violencia que genera (Ochoa et al., 2011). La mayoría de la muestra no tiene ningún tipo de enfermedad física. Un elevado porcentaje está soltero, más de la mitad realiza actividades físicas como el senderismo, e indica que esto les beneficia en la reducción de sus síntomas (Francisco et al., 2005).

La mitad de la muestra ha consumido sustancias psicoactivas siendo el alcohol junto con la marihuana las sustancias más consumidas, aunque prevalece el consumo de tóxicos más nocivos en la muestra experimental. Con respecto a los resultados de la enfermedad, hallamos, siguiendo la clasificación de Crow (1997), que más de la mitad de la muestra $(56,7 \%)$ tiene sintomatología positiva. Se confirma en nuestro estudio que la esquizofrenia que cursa con sintomatología positiva es más frecuente en los estudios de esquizofrenia (APA, 2013; Crow, 1997).

En segundo lugar, los resultados de las pruebas de pragmática ponen de manifiesto la existencia de un déficit significativo en el grupo experimental, especialmente en lo que respecta a las habilidades cognitivas que dan soporte a la habilidad pragmática específica y a la coherencia discursiva. Se observa también que, aunque la pragmática de base gramatical (única prueba del estudio que evalúa la morfosintaxis) se encuentra afectada, lo está en menor medida que el resto de áreas de la pragmática, hecho ya comentado por algunos autores (Covington, 2005; DeLisi, 2001). La estructura sintáctica del discurso de los pacientes del presente estudio se encuentra preservada, a pesar de que su mensaje es redundante, repetitivo y a veces incoherente. Las malas puntuaciones en la evaluación de la pragmática específica, podrían ser consecuencia de las dificultades en la Teoría de la Mente que experimentan estos pacientes (Sprong, Schothorst, Vos, Hox y Van Engeland, 2007).

En cuanto al discurso oral analizado con la lámina del Test de Boston (Goodglass y Kaplan, 1992), podemos confirmar la idea de que el lenguaje de las personas con esquizofrenia tiende a la repetición de palabras, presenta dificultad a la hora de encontrar las palabras, así como la sustitución de estas por otras de significados y sonidos parecidos (Belinchón, 1988b; Covington, 2005). Estos resultados concuerdan con estudios similares en los que se le pedía a un grupo control y a personas con esquizofrenia el describir una lámina (Reich y Cutting, 1982).

También se ha supuesto que las personas con esquizofrenia y los sujetos con lesión cerebral tienden a utilizar expresiones metafóricas en sentido literal (Chapman et al., 1964). Por ello se uso el protocolo MEC para refutar esta idea. Algunos estudios como los de Insúa et al., 2001; Martínez-Sánchez et al., 2015; Pascual et al., 2005 indican como estas áreas están afectadas aunque se emplearan otras medidas para su evaluación. Es importante resaltar que esta prueba también evalúa la memoria a corto plazo y la atención sostenida, aspectos cognitivos que se encuentran afectados en la esquizofrenia (Cavieres y Valdebenito, 2005; Pardo, 2005), por lo tanto, estos resultados hay que tomarlos con cierta cautela pues no se puede asegurar 
que el mal rendimiento en ella sea a causa de un déficit únicamente en el lenguaje. En cualquier caso, el déficit de las personas con esquizofrenia no solo se refleja en el lenguaje oral, sino también en el escrito.

En tercer lugar, la prosodia se encuentra también alterada, tanto en lo referido a sus parámetros acústicos, con frecuencias fundamentales inusitadamente altas en el grupo experimental (tanto para sujetos con sintomatología positiva como negativa), como en lo que respecta a las capacidades comprensivas y expresivas de emociones a través de la entonación (más afectadas en los pacientes con sintomatología negativa).

En nuestro trabajo se ha examinado la comprensión de la prosodia emocional a través de un enunciado con contenido lingüístico emocionalmente neutro. Esta metodología ha sido anteriormente empleada en otros estudios (Hoekert et al., 2007; Pascual et al., 2005) que señalan un déficit en este ámbito en los pacientes con esquizofrenia, observación que se ve confirmada por nuestros resultados.

Ahora bien, nuestros resultados señalan además que la comprensión de la prosodia emocional se encuentra más afectada en pacientes con síntomas negativos, lo que difiere de algunos estudios que indican que no existe diferencia según la sintomatología de los pacientes en la evaluación de la prosodia afectiva expresiva y comprensiva (Rossell y Boundy, 2005).

Existe poca evidencia acerca de las características de la prosodia expresiva de los pacientes diagnosticados de esquizofrenia, pues la mayoría de los estudios que evalúan la expresión de emociones emplean para ello la expresión facial (Kring y Moran, 2008). En nuestro estudio se analizan estas características a través del programa PRAAT. Los parámetros acústicos para la expresión de cada emoción fueron comparados con otros estudios (Esquerra, 2006; Martínez-Sánchez, 2015) comprobando que dichos parámetros en los sujetos con esquizofrenia diferían de los de la población estándar. Los estudios que han examinado la prosodia expresiva obtienen resultados similares a los nuestros (Murphy y Cutting, 1990). Por lo que se refiere a la frecuencia fundamental, Martínez, Felizzola y Matallana (2015) indican que en los pacientes con esquizofrenia se encuentran importantes alteraciones de los elementos suprasegmentales del habla, como una menor variabilidad de la frecuencia fundamental, así como de la intensidad y las pausas en el discurso. En nuestro estudio, se observó significación tanto en la frecuencia fundamental como en la velocidad de habla de todos los sujetos de la muestra, resultados coincidentes con los de otros estudios (Bedwell, Cohen, Trachik, Deptula y Mitchell, 2014; Hoekert et al., 2007; Kring y Moran, 2008).

En cuanto a la comprensión de metáforas los resultados de nuestro trabajo nos permiten afirmar, al igual que otros autores (Covington, 2005; Liddle et al., 2002), que los pacientes con esquizofrenia pueden presentar dificultades en la interpretación no literal de las expresiones lingüísticas, pues una amplia cantidad de sujetos tiene rendimientos por debajo de los baremos de corrección de la prueba. Se confirma así la hipótesis de algunos autores (Chapman et al., 1964), de que los pacientes con esquizofrenia realizarían una interpretación literal de mensajes con doble sentido.

De este modo, se confirma nuestra hipótesis de partida, ya que los resultados muestran que el hecho de cursar con esquizofrenia hace que los sujetos obtengan peores rendimientos en las pruebas de evaluación de la pragmática y la prosodia afectiva que las personas sin trastorno mental. La esquizofrenia cursa con déficit en las áreas analizadas, si bien existe una controversia acerca de su origen, pues algunos 
autores indican que los déficit pragmático y prosódico se producen debido al déficit cognitivo y al trastorno del pensamiento (Cavieres y Valdebenito, 2005; Covington, 2005; Pardo, 2005), mientras que otros los consideran como un déficit primario de la enfermedad (Chapman et al., 1964; Kleist, 1960; Liddle et al., 2002).

En cualquier caso, lo que sí sabemos es que las dificultades lingüísticas, prosódicas y pragmáticas resultan altamente incapacitantes para estos pacientes. De ahí la necesidad de profundizar en el estudio de las dificultades lingüísticas y cognitivas en la esquizofrenia, con el objetivo de llegar a disponer de pruebas de evaluación objetiva de la enfermedad, ya que hasta ahora la mayoría de evaluaciones se basan en pruebas subjetivas que rellenan los pacientes y/o los familiares acerca de cómo se sienten, sobre la sintomatología de la enfermedad, etc. (APA, 2013; National Institute of Health, 1992).

En efecto, el análisis de los fenómenos lingüísticos de manera objetiva puede ayudar a los clínicos a buscar marcadores tempranos de un diagnóstico diferencial basado en el lenguaje como, de hecho, ya se ha planteado en algunos estudios (Figueroa, 2015).

En este sentido, sería también interesante diseñar programas de rehabilitación lingüística desde una perspectiva multidisciplinar con el fin de mejorar la calidad de vida de estos pacientes. Igualmente, es importante seguir definiendo el perfil lingüístico de la esquizofrenia, así como, estudiar si el logopeda, como especialista en comunicación y lenguaje, podría ser el profesional que desarrollase, conjuntamente con otros profesionales, tanto la evaluación como la intervención del déficit lingüístico en este tipo de trastornos mentales, lo que supondría una evolución de la profesión, al adaptar sus conocimientos y habilidades a nuevos contextos (ANECA, 2004; Guijarro-Villar, Martínez-Lorca y González, 2016). Estamos convencidos de que un enfoque interdisciplinar puede aportar profundidad y foco al estudio del complejo campo del lenguaje en la esquizofrenia.

Por último, el presente trabajo tiene una serie de limitaciones. En primer lugar, los test de evaluación lingüística utilizados para esta investigación no están baremados en población con esquizofrenia, por lo tanto, es preciso interpretar los resultados con cautela. En segundo lugar, hubiera sido necesario contar con un grupo control bien emparejado y/o con baremos adecuados para las características sociodemográficas de la muestra en población normal, ya que pueden modificar los resultados de algunas de las pruebas utilizadas. En tercer lugar, el tamaño de la muestra y la localización geográfica de la misma puede considerarse reducida y poco representativa a fin de poder realizar generalizaciones. En cuarto lugar, habría que tener en cuenta que el consumo reiterado de algunos tóxicos como el alcohol puede modificar los resultados de las pruebas pues este aumenta las puntuaciones de los síntomas negativos (Arias, Sánchez y Padín, 2002), así como del cannabis que induce síntomas psicóticos (Arias, 2007).

\section{Bibliografía}

Abusamra,V., Martínez Cuitiño, M., Molina, G. y Ferreres, A. (2006). Evaluación de la comunicación en lesionados derechos: adaptación al español de la batería de Montreal. En XIII Jornadas de Investigación y Segundo Encuentro de Investigadores en Psicología del Mercosur. Universidad de Buenos Aires: Facultad de Psicología. pp. 177-178. Recuperado de: https://www.aacademica.org/000-039/97 
American Psychiatric Association. (2013). Diagnostic and statistical manual of mental disorders (DSM-5). Washington, D.C.: American Psychiatric Publishing.

Andreasen, NC. (1984). The scale for the assessment of positive symptoms (SAPS), and scale for the assesment of negative symptoms (SANS). Iowa City, Iowa: The University of Iowa.

Agencia Nacional de Evaluación de la Calidad y Acreditación. (2004). Libro blanco. Título de Grado en Logopedia. Madrid: ANECA.

Arias, F., Sánchez, S., y Padín, J. J. (2002). Influencia del consumo de drogas en las manifestaciones clínicas de la esquizofrenia. Actas Españolas de Psiquiatría, 30 (2), 65-73.

Arias, F. (2007). Revisión sobre el consumo de cannabis como factor de riesgo de la esquizofrenia. Adicciones, 19 (2), 191-203.

Baddeley, A. D. (1993). Working memory and conscious awareness. En Collins, A.F., Gathercole, S.E., Conway, M.A., y Morris, P.E. (Eds.), Theories of memory (pp. 11-28). Hove, U.K: Lawrence Erlbaum Associates Ltd.

Beaucousin, V., Lacheret, A., Turbelin, M. R., Morel, M., Mazoyer, B. y Tzourio-Mazoyer, N. (2006). FMRI study of emotional speech comprehension. Cerebral cortex, 17 (2), 339-352.

Bedwell, J. S., Cohen, A. S., Trachik, B. J., Deptula, A. E. y Mitchell J. C. (2014). Speech Prosody Abnormalities and Specific Dimensional Schizotypy Features: Are Relationships Limited to Males? The Journal of Nervous and Mental Disease, 202 (10), 745-751. doi: 10.1097/NMD.0000000000000184

Belinchón, M. (1988a). Hacia una caracterización empírica del lenguaje esquizofrénico: de la descripción estructural de los discursos a la reconstrucción de los procesos implicados en la producción verbal desviada. Estudios de Psicología, 9 (33-34), 157-189. doi: 10.1080/02109395.1988.10821520

Belinchón, M. (1988b). Esquizofrenia y lenguaje: una aproximación psicolingüística. Estudios de Psicología, 9 (33-34), 105-144.

Belinchón, M. (1986). Producción verbal y esquizofrenia: Una aproximación psicolingüistica al estudio de las alteraciones del discurso. Tesis Doctoral, Madrid: Universidad Complutense de Madrid.

Benton, AL. y Hamsher K. (1989). Multilingual aplasia examination, $2^{a}$ ed. Iowa City: Department of Neurology and Psychology, The University of Iowa.

Berberian, A., Moraes, G., Gadelha, A., Brietzke, E., Fonseca, A., Scarpato, B., Vicente, M., Seabra, A., Bressan, R. y Lacerda, A. (2016). Is semantic verbal fluency impairment explained by executive function deficits in schizophrenia? Revista Brasileira de Psiquiatria, 38, 121-126. doi:10.1590/1516-4446-2015-1663.

Cavieres, F. y Valdebenito, V. (2005). Funcionamiento cognitivo y calidad de vida en la esquizofrenia. Revista Chilena de Neuro-psiquiatría, 43 (2), 97-108.

Chapman, L. J., Chapman, J. P. y Miller, G. A. (1964). A theory of verbal behavior in schizophrenia. Progress in Experimental Personality Research, 72, 49-77.

Covington, M. A., He, C., Brown, C., Naci, L., McClain, J. T., Fjordbak, B. S. y Brown, J. (2005). Schizophrenia and the structure of language: the linguist's view. Schizophrenia Research, 77 (1), 85-98.

Crow, T.J. (1997). Schizophrenia as failure of hemispheric dominance for language. Trends in Neurosciences, 20 (8), 339-343. doi:10.1016/S0166-2236(97)01071-0.

DeLisi, L.E. (2001). Speech disorder in schizophrenia: Review of the literature and exploration of its relation to the uniquely human capacity for language. Schizophrenia Bulletin, 27 (3), 481-496.

Durán, E. y Figueroa, A. (2009). Sobre el déficit pragmático en la utilización de pares adyacentes por pacientes esquizofrénicos crónicos y de primer brote. Revista Chilena de 
Neuro-psiquiatría, 47 (4), 259-270.

Esquerra, I. (2006). Síntesis de habla emocional por selección de unidades. En Buera, L., Lleida, E., Miguel, A. y Ortega, A. (Eds.), Libro de Actas de las IV Jornadas en Tecnología del Habla, (pp. 161-165). Zaragoza: Universidad de Zaragoza.

Estabrooks, N. y Albert, M. (2004). Manual of aphasia and aphasia therapy. USA: ED-Publishing.

Fairbanks, H. (1944). The quantitative differentiation of samples of spoken language. Psychological Monographs, 56 (2), 17-28. doi:10.1037/h0093509

Faber, R., Abrams, R., Taylor, M. A., Kasprison, A., Morris, C. y Weisz, R. (1983). Comparison of schizophrenic patients with formal thought disorder and neurologically impaired patients with aphasia. The American Journal of Psychiatry, 140 (10), 1348-1351. doi: 10.1176/ajp.140.10.1348

Faber, R. y Reichstein, M. B. (1981). Language dysfunction in schizophrenia. The British Journal of Psychiatry, 139 (6), 519-522.

Francisco, V., Hervás, R. y Gervás, P. (2005). Análisis y síntesis de expression emocional en cuentos leidos en voz alta. Pensamiento del Lenguaje Natural, 35, 293-300.

Fernández-Urquiza, M., Díaz, F., Moreno, V., Lázaro, M. y Simón, T. (2015). Protocolo Rápido de Evaluación Pragmática Revisado. Valencia: Universidad de Valencia.

Figueroa Barra, A. (2015). Análisis pragmalingüistico de los marcadores de coherencia en el discurso de sujetos con esquizofrenia crónica y de primer episodio. Tesis Doctoral, Valladolid: Universidad de Valladolid.

Frostig, J. P. (1940). Clinical observations in the insulin treatment of schizophrenia: Preliminary report. American Journal of Psychiatry, 96 (5), 1167-1190.

Gallardo-Paúls, B. y Hernández Sacristán, C. (2013). Lingüística Clínica. Un enfoque funcional sobre las alteraciones del lenguaje. Madrid: Arco/Libros.

Gómez, A. P. (1976). Pensamiento y lenguaje en la esquizofrenia: revisión de un problema complejo y confuso. Revista Colombiana de Psicología, 21 (1-2), 83-93.

Goodglass, H. y Kaplan, E. (1992). Evaluación de la afasia y de tratornos relacionados. Madrid: Médica Panamericana.

Guijarro-Villar, C., Martínez-Lorca, M. y González, J. (2016). La importancia de la estimulación intrauterina: Nuevos contenidos y retos en los programas de educación maternal. Enfermería Clínica, 26, 262-263. doi: 10.1016/j.enfcli.2015.11.005

Hoekert, M., Kahn, R. S., Pijnenborg, M., y Aleman, A. (2007). Impaired recognition and expression of emotional prosody in schizophrenia: review and meta-analysis. Schizophrenia Research, 96 (1-3), 135-45. doi: 10.1016/j.schres.2007.07.023.

Insúa, P., Grijalvo, J. y Huici, P. (2001). Alteraciones del lenguaje en la esquizofrenia: Síntomas clínicos y medidas psicolingüísticas. Revista de la Asociación Española de Neuropsiquiatría, 21 (78), 27-50.

Knapp, M., Mangalore, R. y Simon, J. (2004). The global costs of schizophrenia. Schizophrenia Bulletin, 30 (2), 279-93.

Kleist, K. (1960). Schizophrenic symptoms and cerebral pathology. Journal of Mental Science, 106, 246-255.

Kring, A. M. y Moran, E. K. (2008). Emotional response deficits in schizophrenia: insights from affective science. Schizophrenia Bulletin, 34 (5), 819-834

Kubicki M., McCarley, R. W., Nestor, P.G., Huh, T., Kikinis, R., Shenton, M.E. y Wible, C.G. (2003). An fMRI study of semantic processing in men with schizophrenia. Neuroimage, 20 (4), 1923-1933.

Leentjens, A. F., Wielaert, S. M., van Harskamp, F. y Wilmink F. W. (1998). Disturbances 
of affective prosody in patients with schizophrenia: a cross sectional study. Journal of Neurology, Neurosurgery \& Psychiatry, 64 (3), 375-378.

LeDoux, J. y Bernal, I. M. (1999). El cerebro emocional. Barcelona: Planeta.

Liddle, P., Ngan, E.T., Caissie, S.L., Anderson, C.M., Bates, A.T., Quested, D.J., White, R., y Weg, R. (2002). Thought and Language Index: an instrument for assessing thought and language in schizophrenia. The British Journal of Psychiatry, 181 (4), 326-330.

Mondragón-Maya, A., Ramos-Mastache, D., Román, P. y Yáñez Téllez, G. (2017). Cognición social en esquizofrenia, familiares no afectados e individuos en riesgo ultra-alto de psicosis: ¿Qué sabemos actualmente? Actas Españolas de Psiquiatría 45 (5), 218-26.

Martínez, A., Felizzola Donado, C. A., y Matallana Eslava, D. L. (2015). Valoración de prosodia espontánea afectiva y análisis de discurso en pacientes con esquizofrenia y demencia frontotemporal (DFT) variante lingüística. Revista Colombiana de Psiquiatría, 44 (1), 13-19.

Martínez-Sánchez, F., Muela-Martínez, J.A., Cortés-Soto, P., Meilán, J.J.G., Ferrándiz, J.A.V., Caparrós, A.E., y Valverde, I.M.P. (2015). Can the acoustic analysis of expressive prosody discriminate schizophrenia? The Spanish Journal of Psychology, 18, E86. doi:10.1017/sjp.2015.85.

Mesholam-Gately, R., Giuliano, A., Goff, K., Faraone, S. y Seidman, L. (2009). Neurocognition in first-episode schizophrenia: a meta-analytic review. Neuropsychology, 23 (3), 315-336.

Murphy, D. y Cutting J. (1990). Prosodic comprehension and expression in schizophrenia. Journal of Neurology, Neurosurgery \& Psychiatry, 53 (9), 727-730.

Napal, O., Ojeda, N., Sánchez, P., Elizagárate, E., Peña, J., Ezcurra, J. y Gutiérrez, M. (2012). Curso evolutivo de la esquizofrenia y su impacto en la cognición: una revisión de la literatura. Actas Españolas de Psiquiatría, 40 (4), 198-220.

National Institute of Health. NHS Confederation. (1992). Early intervention in psychosis services. Briefing, 219. www.iris-initiative.org.uk/silo/files/nhs-confederation-briefing-on-early-intervention-in-psychosis.pdf.

Ochoa, S., Martínez, F., Ribas, M., García-Franco, M., López, E., Villellas, R., Arenas, O., Álvarez, I., Cunyat, C., Vilamala, S., Autonell, J., Lobo, E. y Haro, J.M. (2011). Estudio cualitativo sobre la autopercepción del estigma social en personas con esquizofrenia. Revista de la Asociación Española de Neuropsiquiatría, 31 (3), 477-489. doi: 10.4321/ S0211-57352011000300006

OMS (2016). Guía de Intervención para los trastornos mentales, neurológicos y por uso de sustancias en el nivel de atención de la salud no especializada. Recuperado de: http:// www.who.int/mental_health/publications/mhGAP_intervention_guide/es.

Pardo, V. (2005). Trastornos cognitivos en la esquizofrenia I. Estudios cognitivos en pacientes esquizofrénicos: puesta al día. Revista de Psiquiatría Uruguaya, 69 (1), 71-83.

Pascual, S., Brisa, S., Castillón, J.J., Abadía, Mª J. y Tejedor, Mª C. (2005). Prosodia afectiva y reconocimiento facial y verbal de la emoción en la esquizofrenia. Revista de Psiquiatria de la Facultad de Medicina de Barna, 32 (4), 179-183.

Perkins, M. y Howard, S. (2011). Clinical linguistics. En Simpson, J. (Ed.), The Routledge Handbook of Applied Linguistics (pp.111-123). New York: Routledge.

Reich, S. y Cutting, J. (1982). Picture perception and abstract thought in schizophrenia. Psychological Medicine, 12 (1), 91-96.

Rodríguez, A., Lázaro, P., Montoya, N., Blanco, J.M., Bernadas, D., Oliver, J.M. y Longhi, L. (1998). El habla emocionada en la comunicación audiovisual: una nueva metodología de análisis. Comunicación \& Cultura, 5-6, 59-71.

Rochester, A.R. (1978). Are language disorders in acute schizophrenia actually information processing problems? Journal of Psychiatric Research, 14, 275-283. 
Rossell, S. L. y Boundy, C. L. (2005). Are auditory-verbal hallucinations associated with auditory affective processing deficits? Schizophrenia Research, 78 (1), 95-106.

Ritsner, M.S. y Gottesman, I.I. (2011). The schizophrenia construct after 100 years of challenges. En Ritsner, M. (Ed.), Handbook of Schizophrenia Spectrum Disorders vol. 1. (pp. 1-44). London: Springer.

Salavera, C. y Puyuelo, M. (2010) Aspectos semánticos y pragmáticos en personas con esquizofrenia. Revista de Logopedia, Foniatría y Audiología, 30 (2), 84-93.

Scott, A. (1983). Speech therapy for Parkinson disease. Journal of Neurology, Neurosurgery \& Psychiatry, 46 (2), 140-144.

Scherer, K. J. (1979). Personality markers in speech. En Scherer, K.R. y Giles, H. (Ed.), Social Markers in Speech (pp. 147-209). Cambridge: Cambridge University Press.

Sprong, M., Schothorst, P., Vos, E. Hox, J. y Van Engeland, H. (2007). Theory of mind in squizofrenia: meta-analysis. British Journal of Psyquiatry, 191, 5-13. doi: 10.1192/bjp. bp. 107.035899

Van Dijk, T.A. (1980). Texto y Contexto. Madrid: Cátedra.

Wrobel, J. (1990). Language and Schizophrenia. Amsterdam: John Benjamins Publishing Company. 